\title{
Application of approximate formulae to analyse the spectral distribution of the light attenuation coefficient
}

\author{
Józef KOPEC ${ }^{\text {a }}$, Barbara PAWLAK ${ }^{\text {b }}$ \\ ${ }^{\text {a }}$ Institute of Mathematics, University of Szczecin, Wielkopolska 15, 70-451 Szczecin, Poland \\ ${ }^{\mathrm{b}}$ Institute of Physics, University of Szczecin, Wielkopolska 15, 70-451 Szczecin, Poland
}

(Received 10/04/96, revised 01/04/97, accepted 12/06/97)

\begin{abstract}
An analysis is made of the spectral distribution curves of the light attenuation coefficient in natural water areas and artificial roily oils in sea water samples under various conditions of salinity and storage time, using the approximate formulae based on the Mie theory. (C) Elsevier, Paris
\end{abstract}

mathematical model / light attenuation coefficient / Mie theory

Résumé - Analyse spectrale du coefficient d'atténuation de la lumière à l'aide de formules approchées. La répartition spectrale du coefficient d'atténuation de la lumière a été analysée dans des eaux naturelles et artificielles, dans des émulsions de pétrole, dans des échantillons différant par la salinité et le temps de conservation. L'analyse utilise des formules approchées fondées sur la théorie de Mie. (C) Elsevier, Paris

modèle mathématique / coefficient d'atténuation de la lumière / théorie de Mie

\section{INTRODUCTION}

The light attenuation coefficient, which constitutes an important parameter for describing the optical properties of a water area from both the physical and biological points of view, is assumed [4] to be the sum of absorption and scattering coefficients, and to depend on the physical structure of the water and the presence or otherwise of different types of contamination. Therefore, it can serve as a sensitive indicator of the content of suspended and diluted substances in the sea [5]. In particular, it is used to investigate the composition and particle sizes of suspensions in sea water $[1,3,8]$, and as an indicator contamination when monitoring waters [16].

The mathematical properties of the attenuation coefficient $c(\lambda)$ have been discussed by Kopelevich and Shifrin [11].

The spectral distribution of the absorption coefficient of natural sea water depends on the absorption spectrum of its different components and on their concentrations. In the absence of strong selective absorption bands, their total effect is usually well described by the exponent function $\mathrm{Ae}^{-\alpha \lambda}$.

The scattering phenomenon is described by the scattering function and the global scattering coefficient. The spectra of scattering coefficients have been examined empirically and analysed theoretically in many papers. Most authors use the Mie theory not only to describe the scattering process, but also to examine all real optical characteristics, i.e. the attenuation coefficient $c$, the absorption coefficient $a$, the scattering coefficient $b$ and the scattering function $\beta_{r}$ for different natural collections of suspensions of biological and mineral origin. The theoretical and experimental properties of the coefficient $c(\lambda)$ for many types of natural water areas were discussed in the paper of Prieur and Sathyendranath [13].

For homogeneous particles of approximately spherical shape, the Mie theory provides an analytical description of the basic characteristics of the scattering with the help 
of series composed of Bessel functions and Legendre polynomials $[7,12,17]$.

The analysis of curves describing the spectral distribution of the light attenuation coefficient in natural water areas and in artificial roily oil emulsions in sea water samples under different conditions of salinity and storage time, using approximate formulae based on the Mie theory, is the subject of the present paper.

\section{METHODOLOGY OF EMPIRICAL RESEARCH}

Experiments involved the preparation of samples of roily oil in fresh sea water at appropriate concentrations, and spectrophotometric analysis after different periods of exposure to natural light. Russian roily oil with a density of $0.879 \mathrm{~g} \mathrm{~cm}^{-3}$ at temperature $288 \mathrm{~K}$ was examined. The physicochemical properties of the crude oil examined were presented by Pawlak [13].

Clean sea water was prepared artificially, according to the Polish norm PN-66/C-065502 PKN 1966, for sea water of type B with salinity 35 and $\mathrm{pH} 8.2$. In order to obtain emulsions, $150 \mathrm{~cm}^{3}$ of synthetic sea water were mixed with $0.09 \mathrm{~cm}^{3}$ of crude oil using an electrically-driven universal shaker of the type WU 3. $\Lambda \mathrm{fter}$ two hours of emulsification, the samples were subjected to spectrophotometric analysis with the aid of a dual-beam "SPECORD UV-VIS" spectrophotometer made by the Carl Zeiss firm in Jena. Spectrophotometric measurements were carried out for wavelengths from $260 \mathrm{~nm}$ to $800 \mathrm{~nm}$. Quartz dishes with an optical path length of $5 \mathrm{~cm}$ were placed in the SPECORD at the maximum possible distance from the photometer entrance gap in order to ensure the conditions necessary for measurement of the light attenuation coefficient in absorbing and scattering media [4]. A second, comparative dish was filled with synthetic oceanic water identical to that used for preparation of the emulsion. The differential measurement of light transmission permits determination of the difference between attenuation coefficients for the emulsion $c(\lambda)$, i.e. the determination of the value of $c(\lambda)-c_{\mathrm{w}}(\lambda)$.

Examinations of the light attenuation coefficient were also performed for the waters of the Swina River mouth, using the same SPECORD UV-VIS and in the same wavelength range $(260-800 \mathrm{~nm})$. Distilled water was the comparative medium in these measurements.

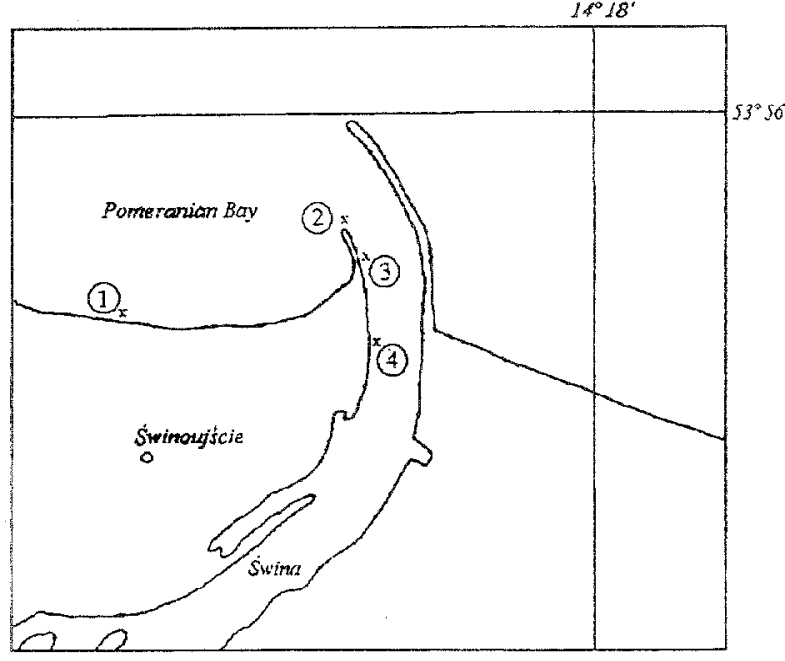

Map 1. Sampling stations: 1 - beach at Swinoujscie (Poland) - seawater; 2 - Swinoujscie windmill - sea water; 3 - the mouth of Swina River - river water; 4 - the mouth of Swina River 2 - river water.

\section{APPROXIMATE FORMULAE}

In the Mie theory, the light scattering rormula is given by:

$b_{r}=2 \pi \int_{r_{\min }}^{r_{\max }} Q_{r^{r^{2}}} N(r) d r$

where

$Q_{r}=\frac{2}{x^{2}} \sum_{k=1}^{\infty}(2 n+1)\left(\operatorname{Re} a_{n}+\operatorname{Re} b_{n}\right)$

\section{$[4,17]$.}

$Q_{r}$ is (in the case of real refractive index) a scattering cross-section.

$\mathrm{N}(r)$ is a dispersion distribution function for scattering particles and the Mie coefficients $a_{n}, b_{n}$ can be calculated from recurrent relations in which $a_{n}, b_{n}$ are expressed by initial values [10]. For real refractive index, these are simple rational functions of tangent function, and for complex refractive index they also depend on hyperbolic functions. Expanding the tangent function into the series of simple fractions and taking only finite number of terms into account, replacing the series (2) by the finite sum and 
the integral by the mean value (or the Riemann sum), we realise that for weak absorption of particles by the material $(\operatorname{Ren} \approx 0$ ), the spectral distribution of the scattering coefficient can be quite well approximated by the rational function of real arguments $\mathrm{x}$ and $\mathrm{y}=\mathrm{n} \mathrm{x}$. If its poles are not in the measured interval, then the form of the scattering coefficient in the interval will be determined by the pole closest to the measured interval, i.e. the fraction $B /\left(\lambda / \lambda_{0}\right)^{p}$. The attenuation coefficient is the sum of the absorption and scattering coefficients, hence assuming that absorption is determined mainly by the first component, we can take

$c(\lambda)=A e^{-\alpha \lambda}+\frac{B}{(\lambda+\beta)^{p}}$,

$p$ is integer. A similar formula was used in [6].

For $\mathrm{p}=1, \alpha>0, \lambda \geq 0$, the form of the curve (3) depends essentially on the signs of the coefficients $A$ and $B$. It can be a monotone, convex curve. It can possess an extreme, zeros and a perpendicular asymptote. Many simplifications were performed while deriving formula (3); hence its applicability remains subject to empirical tests.

Figure 1 presents a diagram of spectral distribution for roily vil in sea water obtained for the data given in table I. The constants $\alpha=0.00541796, \beta=-151.936$, $A=-108.932, B=4102.156$ were found using the least square method. The sum of the differences between the measured and calculated values squared is $H=0.6736$.

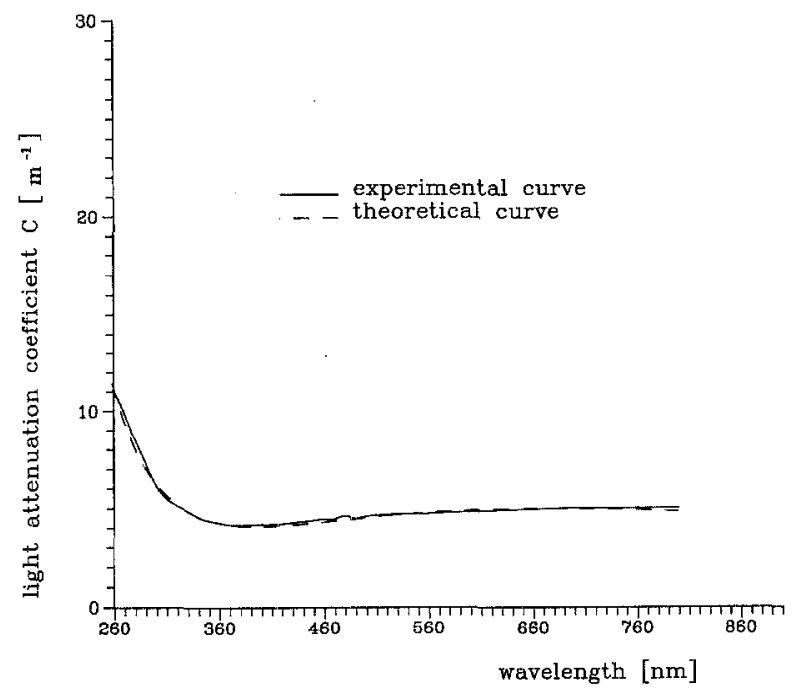

Figure 1. Spectral distribution for roily oil in sea water (theoretical and experimental, corresponding to the data given in table $I$ ).
Table I. Experimental $\left(c_{m}\right)$ and theoretical $\left(c_{t}\right)$ values of the light attenuation coefficient for wavelengths in the $260-800 \mathrm{~nm}$ range.

\begin{tabular}{crrrrr}
\hline$\lambda, \mathbf{n m}$ & $\mathbf{c}_{\mathbf{m}}, \mathbf{m}^{-1}$ & $\mathbf{c}_{\mathbf{t}}, \mathbf{m}^{-\mathbf{1}}$ & $\lambda, \mathbf{n m}$ & $\mathbf{c}_{\mathbf{m}}, \mathbf{m}^{-1}$ & $\mathbf{c}_{\mathbf{t}}, \mathbf{m}^{\mathbf{- 1}}$ \\
\hline 260 & 11.12 & 11.50 & 410 & 4.20 & 4.16 \\
270 & 10.00 & 9.68 & 420 & 4.22 & 4.18 \\
280 & 8.65 & 8.29 & 430 & 4.28 & 4.22 \\
290 & 7.47 & 7.22 & 440 & 4.34 & 4.26 \\
300 & 6.25 & 6.40 & 450 & 4.39 & 4.31 \\
310 & 5.53 & 5.77 & 460 & 4.45 & 4.36 \\
320 & 5.15 & 5.29 & 470 & 4.46 & 4.42 \\
330 & 4.88 & 4.92 & 480 & 4.61 & 4.47 \\
340 & 4.56 & 4.66 & 490 & 4.50 & 4.52 \\
350 & 4.37 & 4.46 & 500 & 4.58 & 4.58 \\
360 & 4.28 & 4.32 & 550 & 4.71 & 4.81 \\
370 & 4.17 & 4.23 & 600 & 4.82 & 4.96 \\
380 & 4.16 & 4.17 & 650 & 4.93 & 5.04 \\
390 & 4.18 & 4.15 & 700 & 5.01 & 5.05 \\
400 & 4.19 & 4.14 & 750 & 5.02 & 5.00 \\
& & & 800 & 5.02 & 4.91 \\
\hline
\end{tabular}

The extremes in the measuring range are $\lambda_{1}=397.3$; $\lambda_{2}=680.7$. Consecutive columns show wavelengths $\lambda$ $(\mathrm{nm})$, measured and calculated value $\left(c_{m}\right.$ and $c_{t}$ respectively) of the attenuation coefficient.

From the above data and from the diagram, it follows that the theoretical curve approximates very well the experimental data and the position of the minimum and maximum. Other usually applied curves give a much worse approximation. For the best polynomial of the 3rd degree (possessing four free parameters) and for the data from table I, the sum of the squared deviations is $\mathrm{H}=14.8$, but for $98 \%$ of the experimental curves for emulsion and for the function (3) we have $\mathrm{H} \leq 3.2$. For $37 \%$ of the curves we have $\mathrm{H}<1$. The relation between the accuracy of the approximation and the measuring error is given in the next section.

\section{STATISTICAL ANALYSIS}

We assume that random variables describing measured results have a form

$x_{i}=F_{i}+e_{i}(i=1, \ldots, n), F_{i}=f^{\prime}\left(\Theta, \lambda_{i}\right)$,

$\Theta=\left[\Theta_{1}, \ldots, \Theta_{p}\right]$ is a vector of parameters, $\boldsymbol{e}$ is a random vector; $E(\boldsymbol{e})=0, \operatorname{cov} \boldsymbol{e}=\sigma^{2} \boldsymbol{I}, \sigma$ depends neither on $i$ nor on $\Theta$. Then the statistics determined by the system of normal equations is the LSM estimator of $\Theta$-vector, and the statistics $\sigma^{2}=H /(n-p)$, where $H=\|\boldsymbol{F}(\boldsymbol{Q})-\boldsymbol{X}\|^{2}$ is an unbiased estimator of variance of the random component. 
In the considered case, $\sigma^{7}=0.674 / 27$, hence the estimated error of a single measurement in the sample is $\sigma=0.178$. Assuming that the $x_{i}$ variables are independent and have distributions $N\left(d_{i}, \sigma_{i}\right)$ where $d_{i}$ is an accurate value of the measured magnitude, a variable

$$
S=\sum_{i=1}^{n}\left[\left(x_{i}-F_{i}\right) / \sigma_{i}\right]^{2}
$$

has a $\chi^{2}$ distribution with $n-p$ degrees of freedom.

With $\sigma$ independent on $i$ the interval $\langle\sqrt{H / q}, \sqrt{H / p}\rangle$ (where $P\left(\chi_{n-p}^{2}<p\right)=\alpha / 2$, and $\left.P\left(\chi_{n-p}^{2} \leq p\right)=1-\alpha / 2\right)$

is a confidence interval at the confidence level $1-\alpha$.

For $\alpha=0.1$ the interval is $\langle\sqrt{H} / 6.333 ; \sqrt{H} / 4.019\rangle$ so in the considered case: $\langle 0.13,0.204\rangle$ which is in agreement with the previous estimation. With $\sigma$ known, the exact value $d_{i}$ belongs to the interval $\left(x_{i}-1.96 \sigma\right.$, $\left.x_{i}+1.96 \sigma\right)$ with the probability 0.95 . In the examined samples we have $H>3$ in only two cases $(3.7 \%)$.

Constancy of a variance for different measured values was verified using 14 samples with 6-28 measurements each with the help of the F test (Fisher - Snedecor). It was confirmed at the significance level $\alpha=0.2$ in all series in which $c_{\text {min }}>5 \mathrm{~m}^{-1}$, and at the level $\alpha=0.05$ in all series in which $c_{\min }>2.5 \mathrm{~m}^{-1}$. If these conditions are not satisfied, the variance must be estimated in different subintervals of the measuring interval. Given the limited stability of samples of products, we regard a relative error of order of $10 \%$ as admissible. Hence, using approximate formulae (with $n=31$ and $p=4$ ) is justified if $\sqrt{H} / c<0 . \sqrt{27}=0.52$. For small measured values $(c<1)$, the estimated relative error can be larger than admissible, approaching as much as $30 \%$. Such measured values are, however, of infrequent occurrence (in a minimum of the function), or in intervals where the attenuation coefficient in emulsions and in natural water areas is practically non-selective. Hence, we believe that approximate formula (3) with $p=I$ can be used for qualitative analysis in the whole measuring range for all $H \leq 3$. In quantitative calculations, the error must be estimated separately in each case.

\section{FSTIMATION OF SPECTRAL DISTRIBUTION STABILITY}

Strong turbulent motions of water cause violent changes in the dispersion distribution of the scattered substance, which then slowly stabilises. In the case of emulsion, the concentration of contamination changes only slightly, but the light attenuation coefficient may alter significantly. For a constant volume of contamination $v$ and for equal size of all drops (in the sample), we have, according to the Mie theory:

$c=N \pi r^{2} Q=\frac{3}{4} v \cdot \frac{1}{r} Q(x), \quad x=2 \pi r / \lambda$

$Q$ is a cross-section for attenuation. The factor $\frac{l}{r} Q(x)$ depends strongly on the radius $r$, but dependence on in the considered interval decreases rapidly with the growth of $r$.

Experimental estimation of the stabilisation time of distribution is difficult under natural conditions. Therefore, we performed spectral distribution measurements on 54 samples of roily oil in synthetic sea water with different salinities and at different temperatures, after different storage times, and on 60 samples of water from the Swina River mouth and Zatoka Pomorska for comparative analysis.

Emulsion storing technology is described in Section 2. The initial concentration of crude oil was chosen in such a way that the input attenuation coefficient of the emulsion would be comparable to the attenuation coefficient of water in strongly contaminated water areas. The change of oil concentration does not affect the process of dispergation and consequently the distribution function of spectral distribution, but affects only the number of particles in the unit volume. As a result, the function $c(\lambda)$ and the constants A, B in formula (3) will be multiplied by a constant factor. In order to obtain conditions similar to natural ones, emulsion samples were stored in glass bottles with natural lighting and temperature. We performed the analysis of spectral distribution of light attenuation coefficients of the emulsion for curves smoothed with the help of formula (3) $(p=1)$.

For the examined sample of fresh roily oil in distilled water with the concentration given above, the curve (3) possesses a minimum for $\lambda=463 \mathrm{~nm}$ which is equal to $c_{\text {min }}=23.6 \mathrm{~m}^{-1}$ and an asymptote in a far infrared. Selectivity $\left(c_{\max } c_{\text {min }}\right)$ in the "optical window" $(350-700 \mathrm{~nm})$ is small $\left(0.94 \mathrm{~m}^{-1}\right)$, a decrease in the violet and ultraviolet part of the spectrum is significantly larger $\left(6.5 \mathrm{~m}^{-1}\right.$ in the interval $260-350 \mathrm{~nm}$ ).

After two weeks of storage, the shape of curve (3) is similar, but depending on the conditions of storage the mini- 


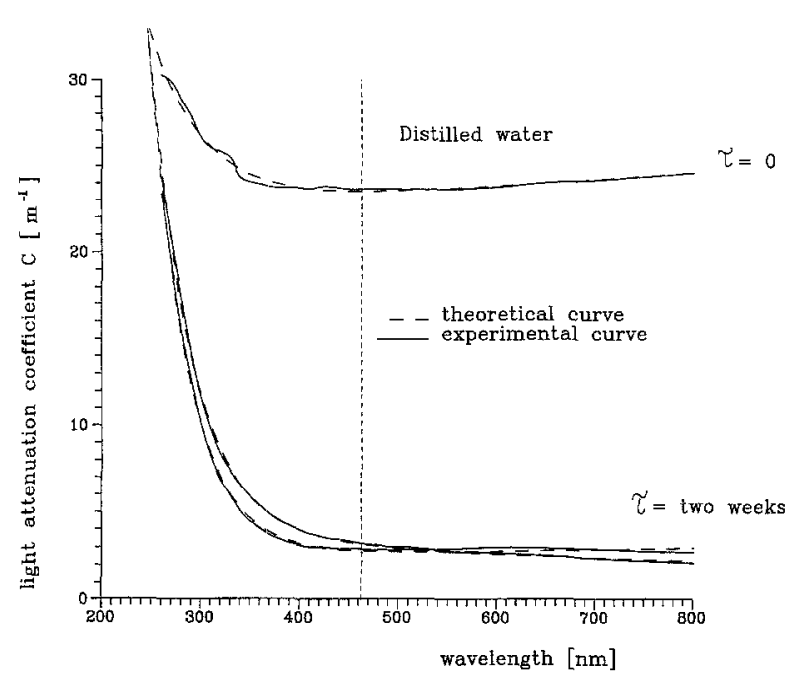

Figure 2. Spectra of the light attenuation coefficient for fresh roily oil in distilled watcr.

mum becomes less distinct or even disappears (the curve is monotone). The attenuation coefficient decreases significantly. For $\lambda=400 \mathrm{~nm}$, it fluctuates between 3 and $8.6 \mathrm{~m}^{-1}$. Selectivity in the interval $400-800 \mathrm{~nm}$ remains small $\left(0.5-1.7 \mathrm{~m}^{-1}\right)$, but the decrease in the interval 260 $400 \mathrm{~nm}$ increases substantially $\left(21-27 \mathrm{~m}^{-1}\right)$. Typical curves are presented in figure 2 ( $\tau$ is storage time). It may be supposed that the main reason for changes in the spectral distribution is a contamination mass loss (outflow to the surface, photodegradation of the oil fall on the bottom) and a related change in drop size.

For salt water, the shape of the spectral distribution curve is different. Figure 3 presents shapes of smoothed curves for fresh samples at the same temperature, with the same crude oil concentration and different salinity.

For a salinity range of 5-30, all curves are of the same type. They possess a minimum in the interval $350-400$ $\mathrm{nm}$, a maximum at $\lambda \approx 650 \mathrm{~nm}$ and an asymptote in the ultraviolet part of the spectrum. This confirms (by extrapolation) the existence of a strong selective attenuation band in ultraviolet and justifies the necessity of examining of oil emulsions in this spectrum range. In the infrared, we observe a slow decrease of the attenuation coefficient down to the value corresponding to water with the same salinity. With a salinity of 5 throughout the measuring interval, there appears to be a decrease (by several orders of magnitude) of the attenuation coefficient in the infrared as compared to the observed one for distilled water and with the same concentration of crude oil. It

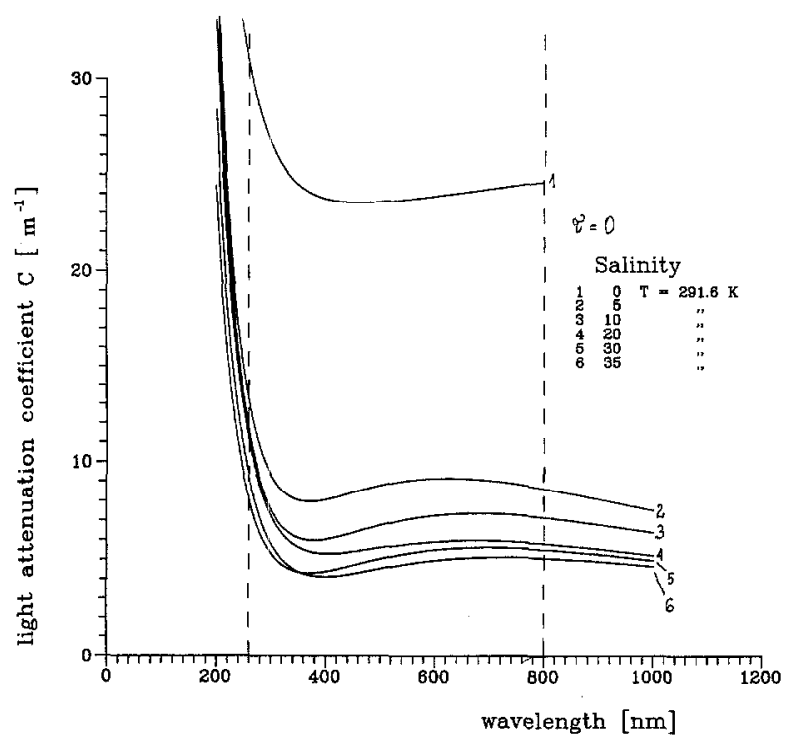

Figure 3. Spectra of the light attenuation coefficient for fresh samples with different salinity.

decreases further with the growth of salinity, but at a remarkably slower rate. Hence, we may conclude that even small salinity (5) causes an essential change in the dispergation mechanism, which alters little with further (even by several orders of magnitude) growth of salinity.

The time factor, stabilisation conditions and a form of stable (smoothed) distribution were examined for two

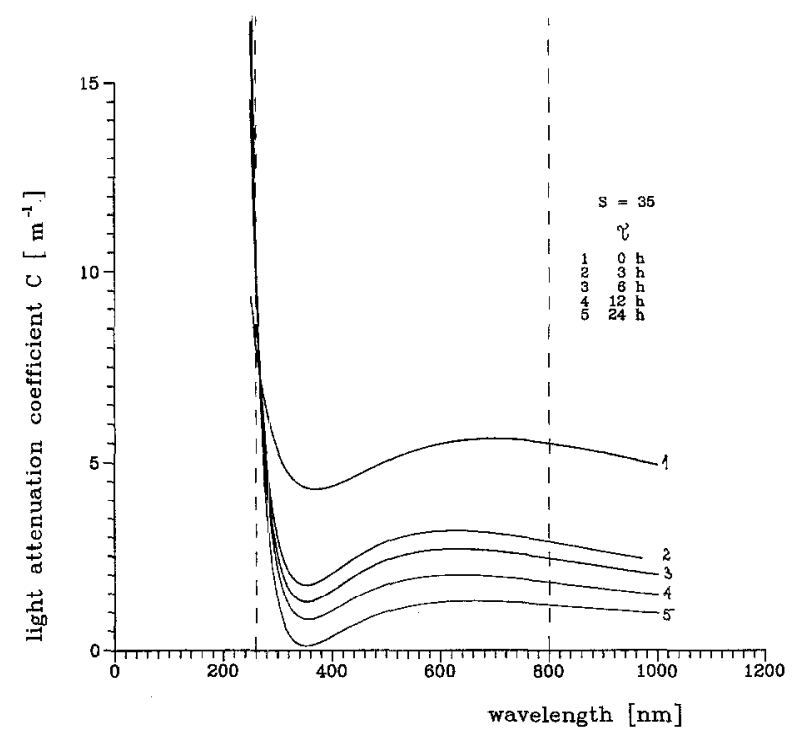

Figure 4. Relationship between the light attenuation coefficient and the storage time of samples. 
Table II. Extreme of the light attenuation coefficient, asymptote and slope of the curve for the temperatures $283 \mathrm{~K}$ and $288 \mathrm{~K}$.

\begin{tabular}{lcccccccc}
\hline Storing time & \multicolumn{3}{c}{ temp. 283 K } & \multicolumn{3}{c}{ temp. 288 K } \\
\hline & $\max$ & $\min$ & $\lambda_{\mathrm{a}}$ & $\mathrm{f}^{\prime}(260)$ & $\max$ & $\min$ & $\lambda_{\mathrm{a}}$ & $\mathrm{f}^{\prime}(260)$ \\
$0 \mathrm{~h}$ & 14.5 & 13.8 & 73.6 & -0.140 & 5.6 & 4.3 & -120 & -0.111 \\
$3 \mathrm{~h}$ & 8.4 & 7.3 & 112 & -0.153 & 3.2 & 1.7 & -198 & -0.350 \\
$6 \mathrm{~h}$ & 5.8 & 4.36 & 138 & -0.190 & 2.7 & 1.3 & -204 & -0.380 \\
$12 \mathrm{~h}$ & 5.1 & 4.17 & 152 & -0.210 & 2.0 & 0.83 & -215 & -0.466 \\
$24 \mathrm{~h}$ & & & & & 1.3 & 0.13 & -226 & -0.593 \\
$72 \mathrm{~h}$ & & & & & 0.8 & 0.5 & -244 & -1.47 \\
\hline
\end{tabular}

particularly interesting values of salinity: 35 (oceanic waters), and 5 (Swina River mouth and Zatoka Pomorska waters). For a salinity of 35 and short storage times $\tau \leq 72 \mathrm{~h}$, the spectral distribution curves are of the same type as for fresh samples with salinity of 5-35 (figure 4).

All are characterised by the large growth of the attenuation coefficient in the ultraviolet part of the spectrum, by the existence of a minimum in the interval $350-405 \mathrm{~nm}$, and a maximum in the interval $570-720 \mathrm{~nm}$, with a slow decrease in the infrared. With the increase in storage time at constant temperature, the extreme of the curves becomes smaller, an asymptote in the ultraviolet shifts to longer waves, and the slope of the curve $\left(f^{\prime}\right)$ increases at the beginning of the measuring interval. At higher temperatures the extremes of the attenuation coefficient are lower, the shift of the asymptote and the slope of the curve are greater for the same storage times. This is illustrated in table II.

After two and three weeks of storage the smoothed curves are monotone for half of the examined samples. The remaining samples still possess two slightly different extremes for $\lambda>550 \mathrm{~nm}$ or, exceptionally, a single minimum.

Hence, we may conclude that for stabilised distributions, the smoothed curve is monotone and convex, but this distribution may not be reached (with the salinity of 35) even after three weeks of storage.

For the fresh sample with a salinity of 5 , a distinct minimum $\lambda \approx 400 \mathrm{~nm}$ and a maximum in the red part of the spectrum appears. After two weeks of storage all smoothed curves are monotone and convex (figure 5).

With this salinity, the distribution is monotone and convex, hence it can be regarded as a stabilised one. Two weeks of storage are sufficient to reach this state. Even low salinity (5) causes an essential change not only in the dispergation mechanism but also in the stabilisation conditions of the spectral distribution.

\section{SPECTRAL DISTRIBUTIONS IN WATER AREAS}

Sixty samples from the mouth of the Swina River and from the Pomerania Bay (Zatoka Pomorska) were examined. Samples were collected every two weeks from 10 January to 27 June 1988 from the surface at four points illustrated on Map 1, and then analysed using SPECORD M-40, in a manner similar to the emulsion (Section 2).

The measured values of the light attenuation coefficient varied significantly from $70.3 \mathrm{~m}^{-1}$ (in the ultraviolet) to $0.02 \mathrm{~m}^{-1}$ (in the infrared).

The mean sum of the squared deviations $H$ of measured and calculated - from formula (3) - values is greater than for the emulsion and fluctuates between 0.45 and 39 . But the number of measurements in different series (40) was greater than for the emulsion (31), hence an error of a sin-

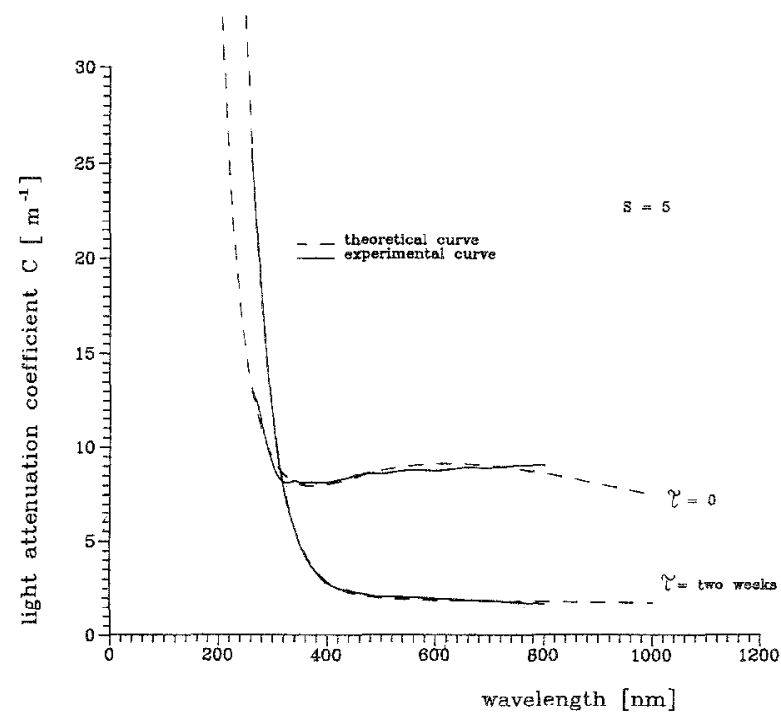

Figure 5. Spectral distribution curves (theoretical and experimental) for samples with salinity $S=5$ after two weeks of storage (2); (1) fresh sample. 
gle measurement is smaller for the same value of $H$. In particular for $H$ equal to 4,9 , and 16 estimated values of $\sigma$ are $0.33,0.5$ and 0.67 respectively. For large mea sured values, a relative error of the calculated value (with respect to the measured one) can be small even for $H>16$. For small measured values $(<0.1)$ appearing in some series in the red and infrared part of the spectrum, the relative error can exceed $10 \%$, in spite of a small mean and absolute error. For measured values $<0.05$, even with a small mean and absolute error, calculated negative values that are not observable in samples may appear. One reason for this, apart from the approximation error, can be the use of distilled water (as a comparative medium) rather than sea water with the same composition as the sample. The necessity of using this medium stemmed from the large fluctuations of salinity due to the variable inflow of waters from the Szczecin Lagoon (Zalew Szczeciñski) with significantly lower salinity into the examined water area and by different contents of dissolved substances selectively absorbing light (e.g. because of the existence of phytoplankton). Light attenuation coefficient spectral distribution curves have a shape quite different from that observed in the case of the emulsion.

All smoothed curves can be divided into two groups:

(I) monotone convex curves (78 \%), for which both constants A, B in formula (3) are positive, and which correspond to stabilised distributions in the case of emulsion; (II) curves with a minimum in the measuring interval, for which the constants A and B have opposite signs. Apart from one case there are, however, no curves with two extremes characteristic for emulsion in a non-stability phase. In this group ( $22 \%$ ), all curves correspond to small measured values, negative and minimal calculated values and a relatively large mean error. In this group of samples, formula (3) gives a worse approximation or may even not be applicable. Essential improvement of the accuracy of the approximation can be obtained by using the formula:

$c(\lambda)=A e^{-\alpha \lambda}+\frac{B}{\lambda+\beta}+c$

New constants can be calculated in the usual manner (the sum of the squared deviations LSM). If again min $c(\lambda)<0$, then the value of the constant $c$ must be changed. This can cause an increase of $\mathrm{H}$, however, for curves from group II application of the formula (4) led to a remarkable decrease in the value in $90 \%$ of cases.
When the constants calculated from formulae (3) and (4) differ significantly one from the other, then the decrease of $\mathrm{H}$ is particularly large. Some decrease in the mean error appeared only for curves possessing a single sharp extreme (peak). Such curves do not exist in the case of emulsion.

While applying formulae (3) and (4) for smoothing out the curves, the distribution of the sum of the squared deviations is given in table III below:

Table III. Distribution of the sum of the squared deviations.

\begin{tabular}{lcccc}
\hline $\mathbf{H}$ & $<4$ & $<4-9)$ & $<9-16)$ & $\geq 16$ \\
\hline Number of series & 22 & 30 & 6 & 2 \\
\hline
\end{tabular}

It follows that for natural water areas, the accuracy of approximation of the experimental curves by formula (3), even applying the correction (4), is worse than for the emulsion. This could be due to many factors, e. g. presence of colour dissolved substances, significant amount of phytoplankton, matter non-homogeneity of scattered particles, deviations from sphericity. Estimation of the influence of different factors is not possible without the aid of applied analytical and measuring techniques.

Figure 6 presents exemplary measuring curves smoothed out with the help of formulae (3) or (4).

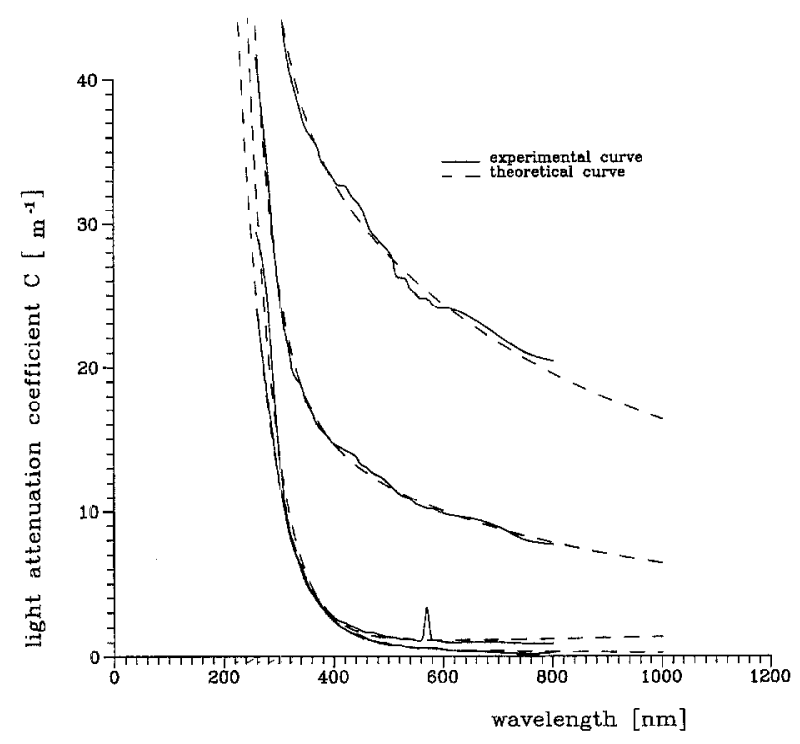

Figure 6. Illustration of approximation accuracy for different values of $\mathrm{H}$ 
It follows that for minimum measured values ( $>2$ ) which are not too small, the smoothed curves are in quite good agreement with experimental curves, even for $H>16$. Recalling that the number of measurements for the natural area water (40) is greater than for the emulsion (31), and that for the curves from group II, the large value of $H$ is caused mainly by the appearance of single peaks, we can accept that approximations with the help of formulae (3) or (4) in approximate categories of curves are sufficiently good for qualitative anlalysis. Therefore, approximations based on the Mie theory, even with substantial mathematical simplifications, may be applied to the analysis of spectral distribution in natural water areas with salinity of about 5, despite the fact that the assumptions of the theory are satisfied with a much lower degree of accuracy.

From the measurements performed on waters of the Odra River in the area of the Szczecin agglomeration, it may be concluded that these formulae cannot be applied for the same purpose in intensively contaminated fresh waters [9].

\section{REFERENCES}

[1] Baker E.T., Lavelle J.W., The effect of particle size on the light attenuation coefficient in natural suspensions, J. Geophys. Res. C89 (1984) 8197-8204.

[2] Bartoszewicz J., Lectures on Mathematical Statistics, Warszawa, PWN (1989) 205-207.

[3] Bricaud A., Morel A., Light attenuation and scattering by phytoplanktonic cells; a theoretical modelling; Appl. Opt. 25 (1986) $571-580$.

[4] Dera J., Marine Physics, Elsevier, Publ. Ocean Ser. Warszawa, Poland (1992) 120-181.

[5] Dera J., Gosh L., Woźniak B., Experimental study of the composite parts of the light-beam attenuation process in the waters of Gulf of Gdañsk; Oceanologia, Sopot, Poland (1978) 5-26.

[6] Dera J., Pawlak B., Light attenuation in sea water polluted with crude oil; 16 th CRO, Kiel (1988) 340-348

[7] Jerlov N.G., Marine optics; Leningrad "Gidrometeoizdat" (in Russian), (1980) 21-75.

[8] Jonasz M., The effect of nonsphericity of marine particles on light attenuation, J. Geophys. Res. C 13 (1987) 14637-14640.

[9] Kopec J., Pawlak B., Szuman M., Light attenuation in emulsion of crude oil in water; The First Szczecin-Helsinki Seminary physics of the sea; Szczecin, Poland, Conference Proceedings No. 2 (1994) 63-73.
[10] Kopec J., Pawlak B., Morocka I., Mathematical models of light attenuation coefficient changes in waters of the Lower Odra, All - Polish; Conference TERB, Gdynia, Poland, 11 (1993) $18-19$.

[11] Kopelevich O.W., Shifrin K.S., Sovremennyye predstawleniya ob opticheskikh svoistvakh morskoy vody; Optika Okeana i Atmosfery, Nauka, Moskva (1981) 4-55.

[12] Mie G., Beiträge zur Optik trüber Medien, speziell Kolloidalen Metallosungen; Ann. Phys. 25 (1908) 377-445.

[13] Pawlak B., Ph. D. thesis; Agricult. Acad. Szczecin (in Polish), (1985) 6-100.

[14] Polish Norm PN 66/C 065502, Vicarious sea water, Polish Normalizing Committee (1966).

[15] Prieur I., Sathyendranath S., An optical classification of coastal and oceanic waters based on the specific spectral absorption curves of phytoplankton pigments, dissolved organic matter and other particulate materials, Limnol. Oceanogr. 26 , 4 (1981) 671-689.

[16] Topliss B. J., Optical monitoring of coastal waters, Photic depth estimates, Mar. Environ. Res. 4 (1982) 295-308.

[17] Van de Hulst H. C., Light scattering by small particles, Wiley, New York, 1957, $470 \mathrm{p}$. 